\title{
Empagliflozin Ameliorating Effect of on Plasma Triglyceride: An Association with Endothelial Function Recovery in Diabetic Patients with Coronary Artery Disease
}

\author{
Takahiro Sawada $^{1 *}$, Kenzo Uzu${ }^{1}$, Naoko Hashimoto ${ }^{2}$, Tetsuari Onishi ${ }^{1}$, Tomohumi Takaya ${ }^{1}$, Akira Shimane ${ }^{1}$, \\ Yasuyo Taniguchi ${ }^{1}$, Yoshinori Yasaka ${ }^{2}$, Takeshi Ohara ${ }^{2}$, Hiroya Kawai ${ }^{1}$
}

${ }^{1}$ Division of Cardiovascular Medicine, Hyogo Prefectural Himeji Cardiovascular Center, Himeji, Hyogo, Japan; ${ }^{2}$ Division of Diabetes and Endocrinology, Hyogo Prefectural Himeji Cardiovascular Center, Himeji, Hyogo, Japan

\begin{abstract}
Introduction: The mechanisms behind the cardiovascular benefits of Sodium-Glucose Co-Transporter 2 (SGLT2) inhibitors have not been fully clarified. We aimed to identify potential mechanisms underlying the favorable effects of SGLT2 inhibitors on cardiovascular events.

Methods: The 50 diabetic patients with established Coronary Artery Disease (CAD) included in this study analysis were administered $10 \mathrm{mg}$ /day of empagliflozin. Cookie meal testing (carbohydrates: $75 \mathrm{~g}$, fat: $28.5 \mathrm{~g}$ ), endothelial function testing using Flow-Mediated Dilatation (FMD), and body composition evaluation were performed before and after 6 months treatments. Evaluation of changes in \% FMD between treatment periods, and its association with metabolic biomarkers were evaluated.

Results: After the 6 month treatment, body weight and body fat percentage decreased significantly, while body muscle percentage increased significantly. $\mathrm{HbA1C}$ level and fasting and postprandial plasma glucose levels were significantly decreased by treatment. Postprandial insulin secretion was also significantly suppressed and insulin resistance index was significantly decreased. Furthermore, fasting and postprandial triglyceride levels decreased significantly while total ketone body increased significantly after the 6 months treatment. While plasma brain natriuretic peptide level was not changed, C-reactive protein was decreased, and FMD was significantly improved after the 6 months treatment. Multiple regression analysis showed the strongest predictive factor of FMD improvement to be change in fasting and postprandial plasma triglyceride levels.

Conclusion: SGLT2 inhibitors improved multiple metabolic parameters. Of these, reduction in plasma triglyceride was strongly associated with endothelial function recovery in diabetic patients with CAD, and this reduction may be related to the cardiovascular benefit of SGLT2 inhibitors.
\end{abstract}

Keywords: Diabetes mellitus; Sodium-glucose transporter 2 inhibitors; Triglycerides; Endothelial function; Flow mediated dilatation

\section{INTRODUCTION}

Type 2 Diabetes Mellitus (DM), a prevalent disease, is recognized as a major risk factor for Coronary Artery Disease (CAD) [1,2]. Selective inhibitors of Sodium-Glucose Co-Transporter 2 (SGLT2) are glucose lowering agents targeting the kidney, to reduce the reabsorption of glucose and promote urinary glucose excretion, and have been approved for type 2 DM treatment in numerous countries [3]. Previous large studies, Empagliflozin Cardiovascular Outcome Event Trial in Type 2 Diabetes Mellitus Patients (EMPAREG OUTCOME) [4] and the Canagliflozin Cardiovascular Assessment Study (CANVAS) trial [5], demonstrated that SGLT2 inhibitors were the first glucose-lowering agents to reduce both cardiovascular death and high risk of cardiovascular disease in patients with type $2 \mathrm{DM}$.

Correspondence to: Takahiro Sawada, MD, Department of Internal Medicine, Division of Cardiovascular Medicine, Hyogo Prefectural Himeji Cardiovascular Center, 520, Saisho-Kou, Himeji, Hyogo, 670-0981, Japan, Tel: +81-79-293-3131; Fax: +81-79-295-8159; E-mail: taktak319@yahoo.co.jp

Received: March 28, 2019, Accepted: April 26, 2019, Published: May 03, 2019

Citation: Sawada T, Uzu K, Hashimoto N, Onishi T, Takaya T, Shimane A, et al. (2019) Empagliflozin Ameliorating Effect of on Plasma Triglyceride: An Association with Endothelial Function Recovery in Diabetic Patients with Coronary Artery Disease. J Diabetes Metab 10:827. doi: 10.35248/2155 6156.19.10.827.

Copyright: ( $) 2019$ Sawada T, et al. This is an open-access article distributed under the terms of the Creative Commons Attribution License, which permits unrestricted use, distribution, and reproduction in any medium, provided the original author and source are credited. 
Because EMPA-REG OUTCOME and CANVAS trials were not designed to determine the mechanism underlying their results, several explanations for the reduction in cardiovascular death with SGLT2 inhibitors have been proposed and included: improved glycemic and lipid control, reduction in body weight, lowering of blood pressure, and amelioration of albuminuria [6-8]. However, the precise mechanisms behind the cardiovascular benefits of SGLT2 inhibitors have not been fully clarified.

Impaired endothelial function may play an important role in the initiation and progression of atherosclerosis [9]. In addition, endothelial dysfunction is contributes to impaired myocardial perfusion, increased arterial wall stiffness and increased left ventricular after load [10]. The noninvasive parameter, FlowMediated Dilatation (FMD) in the brachial artery, is considered to be the current gold standard surrogate marker of coronary endothelial function and previous study demonstrated that the brachial FMD is a predictor of severity of CAD, coronary plaque vulnerability, left ventricular remodeling in ST-segment elevated myocardial infarction, and of cardiovascular events [11-15].

In this study, we surrogated the brachial FMD as the incidence of cardiovascular events and conducted an exploratory study to identify potential mechanisms underlying the favorable effects of SGLT2 inhibitors on cardiovascular events, focusing on the metabolic effects of SGLT2 inhibitors.

\section{MATERIALS AND METHODS}

\section{Participants}

This study, an open label, single arm, prospective interventional study approved by the Ethics Committee of Hyogo Prefectural Himeji Cardiovascular Center, complied with the Declaration of Helsinki. Informed consent was obtained from all eligible patients before participation.

A total of 56 Japanese type 2 DM patients with documented chronic CAD were recruited and enrolled to this study from our outpatients between April 2017 and March 2018. The inclusion criteria were as follows: 1) SGLT2 inhibitor-naïve type 2 DM patients who had been treated for more than 12 weeks with some anti-diabetic drugs, or diet and exercise; 2) $\mathrm{HbA}_{1 \mathrm{C}}$ (National Glycohemoglobin Standardization Program; NGSP) level of $<8.0 \%$; and 3) Males and females aged $40-90$ years. CAD was defined as stenosis of $>50 \%$ of the diameter of a coronary artery on angiography or on computed tomography coronary angiography; or a history of myocardial infarction, percutaneous coronary intervention, or coronary artery bypass surgery. Type $2 \mathrm{DM}$ was diagnosed according to the Japan Diabetic Society criteria $\left(\mathrm{HbA}_{1 \mathrm{C}} \geq 6.4 \%\right.$, fasting Plasma Glucose $(\mathrm{PG}) \geq 126 \mathrm{mg} / \mathrm{dL}$, and $\mathrm{PG} \geq 200 \mathrm{mg} / \mathrm{dL}$ at $2 \mathrm{~h}$ after the oral glucose tolerance test).

The exclusion criteria were as follows: 1) Patients who, within 12 weeks of enrollment, had started or had had the dose changed, or had been on any concomitant drug, such as anti-diabetic drugs, statins, or anti-hypertensive drugs; 2) Patients treated with insulin or glucagon-like peptide-1 agonists; 3) Patients with type 1 DM or secondary DM; 4) Patients with systemic disease, including hepatic disease, renal disease (serum creatinine levels $>2.0 \mathrm{mg} / \mathrm{dL}$ ), collagen disease, infection, malignancy or acute coronary syndrome; and 5) Patients with implanted permanent pacemakers, implanted cardiac defibrillators or cardiac resynchronization therapy devices.
All patients met, at least once, with a dietician for nutritional guidance, and were encouraged to start and maintain a low-calorie diet and mild-to-moderate exercise levels before study enrollment. At enrollment, we added $10 \mathrm{mg} /$ day of empagliflozin to the regimen, which was maintained for 6 months. At each visit, we enquired from the participants about any adverse events and medication compliance. In addition, all participants were prohibited from changing the dose of concomitant drugs or adding any other drugs, throughout the study period if no adverse effect occurred.

\section{Measurements of the body composition}

A body constituent was measured using InBody770 (InBody Co., LTD, Seoul, Korea) at enrollment and after 6 months of treatment. InBody770 sends very weak alternating current through the body, which the body tissue resists. This machine, InBody770, divides the human body into five cylinders (right and left arms, right and left legs, and trunk), in order to increase the accuracy of the measurement, and to deliver the currents of $50-1000 \mathrm{kHz}[16,17]$. Participants were required to stand on the balance scale on bare feet, and hold the handles of the machine. The output of the measurements, which takes approximately 15 seconds, is printed.

\section{Blood biochemistry}

Blood samples were collected from all patients after an overnight fast and were used to determine PG, $\mathrm{HbA}_{1 \mathrm{C}}, 1,5$-anhydro-glucitol, Immune Reactive Insulin (IRI), C-peptide (CPR), total cholesterol, High-Density Lipoprotein (HDL) cholesterol, Low-Density Lipoprotein (LDL) cholesterol, Triglyceride (TG), remnantlike particle cholesterol (RLP-C) as a marker of postprandial hyperlipidemia, total ketone body fraction, brain natriuretic peptide (BNP) as a marker of heart failure, C-Reactive Protein (CRP) as an inflammatory marker, and Plasminogen Activator Inhibitor type 1 (PAI-1) as a marker of thrombotic diathesis. All biochemical analyses were performed using a commercially available kit. $\mathrm{HbA}_{1 \mathrm{C}}$ levels were measured using high-performance liquid chromatography. IRI, CPR and BNP concentrations were measured using a chemiluminescent enzyme immunoassay. RLP-C was measured using an assay kit (Japan Immunoresearh Laboratories Co., Ltd., Takasaki, Japan). The level of total ketone body fraction was measured by enzyme circulating methods (Kinos Inc., Tokyo, Japan). PAI-1 was measured using a latex photometric immunoassay.

\section{Cookie meal test}

A Cookie Meal Test (CMT) was performed before and 6 month after empagliflozin treatment to evaluate the changing postprandial glucose, insulin, and fat metabolic dynamics. The cookie consisted of carbohydrates (75 g; 85\% flour starch, 15\% maltose), butter fat (28.5 g), and protein $(8 \mathrm{~g})$ with $592 \mathrm{kcal}$ (SARAYA Corp., Osaka, Japan). As previously reported, the CMT is sufficient for providing information regarding glucose intolerance and postprandial hypertriglyceridemia [18].

All participants completed a $12 \mathrm{~h}$ overnight fast before the CMT, was previously described [17]. Briefly, the cookie was ingested with water within a $20 \mathrm{~min}$ period, and blood samples for PG, IRI, CPR and TG measurement were obtained at 0,1 , and $2 \mathrm{~h}$ after the participant had ingested half the volume of the cookie. These values were reported as PG-1h, PG-2h, IRI-1h, IRI-2h, CPR-1h, CPR-2h, TG-1h, and TG-2h, respectively. 
As an index of postprandial hyperglycemia, we calculated the incremental glucose peak ((the maximal incremental increase in PG at any point after meal loading)-(fasting PG)). We also calculated the area under the response curves for PG (AUC-PG) and IRI (AUC-IRI) using the trapezoid rule. The homeostasis model assessment ratio (HOMA-R=fasting IRI $\times$ fasting $P G / 405)$ was used as an index of insulin resistance.

The trapezoid rule was used to calculate the area under the TGresponse curve (AUC-TG).

\section{Measurement of endothelial function}

Before undergoing FMD testing, the participants were instructed to fast for $>12 \mathrm{~h}$ and to abstain from any medications, smoking, alcohol, caffeine, and antioxidant vitamins during that time. All participants rested for at least $15 \mathrm{~min}$ in a seated position in a quiet dark airconditioned room $\left(22-25^{\circ} \mathrm{C}\right)$ before the FMD measurements, as previously described $[12,19]$. In brief, a longitudinal image of the right or left brachial artery was recorded at baseline using highresolution ultrasonography and a $10 \mathrm{MHz}$ linear array transducer probe (UNEX, Nagoya, Japan). A forearm-cuff was inflated for 5 min at $50 \mathrm{mmHg}$ above the systolic blood pressure just prior to FMD measurement. After deflating the cuff, the diastolic diameter of the brachial artery was semi-automatically and continuously recorded for $2 \mathrm{~min}$ using software-equipped instrument that could monitor arterial diameter. The \%FMD was estimated as the percent change in the vessel diameter, which corresponded to the maximum dilatation that was reached during reactive hyperemia divided by the baseline value. Because the \%FMD value is highly dependent on the baseline diameter of the vessel, we compared the baseline diameters and absolute changes in the diameters of the brachial artery for each group. We have also previously confirmed that there is excellent intra- and inter-observed agreement for the \%FMD measurement $[13,19,20]$.

\section{Primary endpoints}

The primary endpoint of this study was the change in \%FMD at enrollment and after 6 month empagliflozin treatment. We also evaluated the association between changes in \%FMD and changes in metabolic biomarkers.

\section{Statistical analysis}

Sample size was determined by power analysis using previously reported data with the following assumptions: Type I error of 0.05 (two-tailed), power of $80 \%$, a $1.0 \%$ difference in \%FMD before and after empagliflozin treatment, and a standard deviation of $2.0 \%$, respectively [21]. Therefore, a minimum of 34 patients is required to yield $80 \%$ power to detect a significant difference between before and after treatment.

All statistical analyses were performed using MedCalc software (Version 9.3; Mariakerke, Belgium), and a two-tailed p-value of $<0.05$ was considered statistically significant.

Continuous variables with normal distribution were reported as mean \pm standard deviation, otherwise, as median and interquartile ranges. Inter-group comparisons of normally distributed data were performed using the unpaired Student's t-test. Mann-Whitney $\mathrm{U}$ test was used if the variables were not normally distributed. Comparison of categorical variables was performed using $\chi^{2}$ test.
Differences in normally distributed biochemical data before and after treatment were compared using the paired t-test; otherwise the Wilcoxon rank-sum test was used. Multivariable regression analyses were used to identify independently associated factors of changes in \%FMD. Biologically plausible factors were included in the original model. Potential factors of interest with a univariate $\mathrm{p}<0.1$ were entered into the multivariate models.

\section{RESULTS}

\section{Study population}

A total of 56 diabetic patients with CAD were enrolled in this study. Of these, 1, 1, and 4 patients had hypoglycemic attack, gastrointestinal disorder, and withdrew their consent before the second CMT, respectively. Therefore, the 6 patients were excluded from the final analyses, leaving 50 patients.

Table 1 shows the baseline characteristics of the participants. Concomitant coronary risk factors were hypertension $(90.0 \%)$, dyslipidemia (98.0\%) and smoking (8.0\%). Combined medications were statins (98.0\%), n-3 polyunsaturated fatty acids $(32.0 \%)$, angiotensin converting enzyme inhibitors/angiotensin-receptor blocker (72.0\%), dipeptidyl peptidase 4 inhibitors $(52.0 \%)$, metformin/thiazolidine (20.0\%), sulfonylurea agent (12.0\%), and alpha glucosidase inhibitors (10.0\%). Notably, all patients had CAD and multiple coronary risk factors ( $\geq 2$ coronary risk factors).

\section{Changes in anthropometric measurements and body composition}

As shown in Table 2, body weight and body mass index were significantly decreased after the 6 month empagliflozin treatment $\left(68.6 \pm 9.9 \mathrm{~kg}\right.$ to $65.3 \pm 9.7 \mathrm{~kg}, \mathrm{p}<0.0001 ; 24.9 \pm 3.0 \mathrm{~kg} / \mathrm{m}^{2}$ to $\left.23.7 \pm 3.1 \mathrm{~kg} / \mathrm{m}^{2}, \mathrm{p}<0.001\right)$. Although the total fat quantity $(18.3$ $\pm 5.1 \mathrm{~kg}$ to $15.9 \pm 5.2 \mathrm{~kg}, \mathrm{p}<0.0001)$, fat percentage $(26.9 \pm 5.7 \%$

Table 1: Baseline characteristics.

\begin{tabular}{cc}
\hline Variables & $\mathbf{n}=50$ \\
\hline Age (y.o) & $66.5 \pm 9.4$ \\
\hline Sex (Male, $\mathrm{n}, \%)$ & $44(88.0 \%)$ \\
\hline Coronary risk factor & $45(90.0 \%)$ \\
\hline Hypertension (n,\%) & $49(98.0 \%)$ \\
\hline Dyslipidemia (n,\%) & $4(8.0 \%)$ \\
\hline Current smoker (n,\%) & $49(98.0 \%)$ \\
\hline Combined drugs & $16(32.0 \%)$ \\
\hline Statins (n,\%) & $20(40.0 \%)$ \\
\hline n-3 polyunsaturated fatty acids (n,\%) & $36(72.0 \%)$ \\
\hline Calcium channel blocker (n,\%) & $26(52.0 \%)$ \\
\hline $\begin{array}{c}\text { Angiotensin converting enzyme inhibitors/ } \\
\text { angiotensin-receptor blocker (n,\%) }\end{array}$ & $10(20.0 \%)$ \\
\hline Dipeptidyl peptidase 4 inhibitors (n,\%) & $6(12.0 \%)$ \\
\hline Metformin / thiazolidine (n,\%) & $5(10.0 \%)$ \\
\hline Sulfonylurea agent (n,\%) & \\
\hline Alpha glucosidase inhibitors (n,\%) & Note: y.o: years old \\
\hline
\end{tabular}


to $24.6 \pm 6.3 \%, \mathrm{p}<0.0001)$, total muscle quantity $(46.9 \pm 7.2 \mathrm{~kg}$ to $45.8 \pm 6.1 \mathrm{~kg}, \mathrm{p}<0.0001)$, and water quantity $(36.5 \pm 5.9 \mathrm{~L}$ to $35.7 \pm 5.4 \mathrm{~L}, \mathrm{p}<0.0001)$ were also decreased significantly, muscle percentage was significantly increased $(69.1 \pm 5.5 \%$ to $71.3 \pm 6.1 \%$, $\mathrm{p}<0.0001)$. Furthermore, the systolic and diastolic blood pressure was significantly decreased during the 6 months.

Table 2: Changes in anthropometric measurements and body compositions.

\begin{tabular}{lccc}
\hline Variable & Before & $\begin{array}{c}\text { After } 6 \\
\text { months }\end{array}$ & p-value \\
\hline Weight $(\mathrm{kg})$ & $68.6 \pm 9.9$ & $65.3 \pm 9.7$ & $<0.0001$ \\
\hline Body mass index $\left(\mathrm{kg} / \mathrm{m}^{2}\right)$ & $24.9 \pm 3.0$ & $23.7 \pm 3.1$ & $<0.0001$ \\
\hline Body fat quantity $(\mathrm{kg})$ & $18.3 \pm 5.1$ & $15.9 \pm 5.2$ & $<0.0001$ \\
\hline Body fat percentage $(\%)$ & $26.9 \pm 5.7$ & $24.6 \pm 6.3$ & $<0.0001$ \\
\hline Muscle quantity $(\mathrm{kg})$ & $46.9 \pm 7.2$ & $45.8 \pm 6.9$ & $<0.0001$ \\
\hline Muscle percentage $(\%)$ & $69.1 \pm 5.5$ & $71.3 \pm 6.1$ & $<0.0001$ \\
\hline Water quantity $(\mathrm{kg})$ & $36.5 \pm 5.9$ & $35.7 \pm 5.4$ & $<0.0001$ \\
\hline Extracellular water & $0.38 \pm 0.02$ & $0.38 \pm 0.01$ & 0.13 \\
\hline
\end{tabular}

Total body water

$\begin{array}{llll}\text { Systolic blood pressure }(\mathrm{mmHg}) & 133.8 \pm 15.1 & 130.6 \pm 14.3 & 0.03\end{array}$

$\begin{array}{llll}\text { Diastolic blood pressure }(\mathrm{mmHg}) & 80.3 \pm 10.3 & 78.4 \pm 9.5 & 0.03\end{array}$

\section{Changes in fasting and postprandial blood chemistry analysis and endothelial function}

The fasting and postprandial blood chemistry data at baseline and at 6 months are shown in Table 3. After 6 months of treatment, $\mathrm{HbA}_{1 \mathrm{C}}$ level, fasting PG, and IRI were significantly decreased; furthermore, a significant reduction in the insulin resistance index (HOMA-R) was observed, and a low 1,5-anhydro-glucitol levels indicated a deleterious effect.

Regarding lipid profiles, fasting TG and remnant-like particle cholesterol exhibited significant reduction, although there were no significant changes in total cholesterol, HDL cholesterol, and LDL cholesterol.

The CMT data at baseline and after 6 months of treatment are shown in Table 4. Postprandial PG (PG-1h and PG-2h), IRI (IRI-1h and IRI-2h), and CPR (CPR-1h and CPR-2h) were all decreased significantly. Consequently, significant reductions in AUC-PG, AUC-IRI, and AUC-CPR were also observed. On the other hand, incremental glucose peak remained unchanged. In a similar fashion, the significant reductions in TG-1h, TG-2h, and AUC-PG were also observed.

Although white blood cell count remained unchanged, significant increases were observed in red blood cell count, hemoglobin, and

Table 3: Changes in blood biochemistry and flow-mediated dilatation.

\begin{tabular}{|c|c|c|c|}
\hline Variable & Before & After 6 months & p-value \\
\hline White blood cell $\left(\times 10^{2} / \mu \mathrm{L}\right)$ & $61.6 \pm 14.6$ & $60.7 \pm 15.4$ & 0.67 \\
\hline Red blood cell $\left(\times 10^{4} / \mu \mathrm{L}\right)$ & $476.2 \pm 36.9$ & $498.0 \pm 38.4$ & $<0.0001$ \\
\hline Hemoglobin $(\mathrm{g} / \mathrm{dL})$ & $14.6 \pm 1.1$ & $15.0 \pm 1.2$ & $<0.0001$ \\
\hline Hematocrit (\%) & $43.3 \pm 2.9$ & $44.8 \pm 3.0$ & 0.0001 \\
\hline Platelet $\left(\times 10^{4} / \mu \mathrm{L}\right)$ & $21.7 \pm 4.6$ & $20.5 \pm 4.5$ & 0.001 \\
\hline Plasma creatinine $(\mathrm{mg} / \mathrm{dL})$ & $0.82 \pm 0.16$ & $0.84 \pm 0.17$ & 0.07 \\
\hline $\mathrm{e}-\mathrm{GFR}(\mathrm{mg} / \mathrm{dL})$ & $73.4 \pm 14.6$ & $71.3 \pm 14.0$ & 0.06 \\
\hline Urine albumin (mg/dL) & $16.8(7.2,52.7)$ & $11.3(7.4,25.1)$ & 0.01 \\
\hline Uric acid $(\mathrm{mg} / \mathrm{dL})$ & $5.4 \pm 1.3$ & $4.7 \pm 1.1$ & $<0.0001$ \\
\hline $\mathrm{HbA}_{1 \mathrm{C}}(\%)$ & $7.0 \pm 0.5$ & $6.6 \pm 0.4$ & $<0.0001$ \\
\hline 1,5-anhydroglucitol $(\mu \mathrm{g} / \mathrm{dL})$ & $11.1 \pm 5.7$ & $2.1 \pm 2.0$ & $<0.0001$ \\
\hline \multicolumn{4}{|c|}{ Plasma glucose level (mg/dL) } \\
\hline Fasting & $129.4 \pm 16.1$ & $114.8 \pm 18.7$ & $<0.0001$ \\
\hline After 1 hour & $238.7 \pm 33.4$ & $217.2 \pm 33.6$ & $<0.0001$ \\
\hline After 2 hours & $235.9 \pm 46.4$ & $213.7 \pm 51.1$ & 0.001 \\
\hline Incremental glucose peak $(\mathrm{mg} / \mathrm{dL})$ & $122.2 \pm 31.1$ & $114.3 \pm 34.4$ & 0.08 \\
\hline \multicolumn{4}{|c|}{ Immune reactive insulin $(\mu \mathrm{U} / \mathrm{mL})$} \\
\hline Fasting & $7.8(4.6,9.5)$ & $5.0(3.2,8.5)$ & $<0.0001$ \\
\hline After 1 hour & $40.9(25.0,60.3)$ & $33.5(18.2,59.8)$ & 0.0005 \\
\hline After 2 hours & $49.2(28.3,79.9)$ & $43.0(23.5,60.5)$ & 0.0006 \\
\hline HOMA-R & $2.4(1.4,3.2)$ & $1.3(0.9,2.3)$ & $<0.0001$ \\
\hline \multirow{2}{*}{ AUC-IRI $(\mu \mathrm{U} / \mathrm{mL})$} & 69 & 60.6 & $<0.0001$ \\
\hline & $(41.6,103.8)$ & $(32.1,92.0)$ & \\
\hline \multicolumn{4}{|c|}{ C peptide $(\mathrm{ng} / \mathrm{mL})$} \\
\hline Fasting & $2.1(1.7,2.6)$ & $1.7(1.4,2.2)$ & 0.0001 \\
\hline After 1 hour & $5.4(4.6,7.7)$ & $4.7(3.8,8.0)$ & 0.11 \\
\hline
\end{tabular}




\begin{tabular}{|c|c|c|c|}
\hline After 2 hours & $8.9(6.3,11.3)$ & $7.9(6.5,11.4)$ & 0.11 \\
\hline Total cholesterol (mg/dL) & $164.9 \pm 25.6$ & $163.6 \pm 29.7$ & 0.62 \\
\hline LDL cholesterol (mg/dL) & $90.6 \pm 23.5$ & $90.8 \pm 24.1$ & 0.92 \\
\hline HDL cholesterol (mg/dL) & $51.1 \pm 19.6$ & $51.1 \pm 9.4$ & 0.99 \\
\hline \multicolumn{4}{|c|}{ Plasma triglyceride $(\mathrm{mg} / \mathrm{dL})$} \\
\hline Fasting & $119.9 \pm 54.9$ & $95.7 \pm 33.5$ & 0.0002 \\
\hline After 1 hour & $142.5 \pm 53.5$ & $122.6 \pm 41.0$ & 0.001 \\
\hline After 2 hours & $184.3 \pm 70.2$ & $153.5 \pm 52.8$ & $<0.0001$ \\
\hline \multirow[t]{2}{*}{ AUC-triglyceride $(\mathrm{mg} / \mathrm{dL})$} & 275.5 & 250.5 & 0.0002 \\
\hline & $(206.0,364.0)$ & $(188.0,295.0)$ & \\
\hline RLP cholesterol (mg/dL) & $5.0(3.3,7.0)$ & $3.8(2.4,4.9)$ & $<0.0001$ \\
\hline $\mathrm{C}$ reactive protein $(\mathrm{mg} / \mathrm{dL})$ & $0.11(0.03,0.22)$ & $0.07(0.03,0.13)$ & 0.003 \\
\hline Brain natriuretic peptide $(\mathrm{pg} / \mathrm{mL})$ & $31.3(14.4,58.6)$ & $20.6(9.2,54.6)$ & 0.37 \\
\hline PAI-1 (ng/mL) & $18.6 \pm 7.8$ & $13.8 \pm 5.0$ & $<0.0001$ \\
\hline \multirow{2}{*}{ Total ketone body $(\mu \mathrm{mol} / \mathrm{L})$} & 89 & 145.5 & 0.0002 \\
\hline & $(61.0,152.0)$ & $(94.0,218.0)$ & \\
\hline \%flow-mediated dilatation (\%) & $3.5(2.4,5.5)$ & $4.7(3.6,5.6)$ & 0.001 \\
\hline Absolute change in diameter $(\mathrm{mm})$ & $0.17 \pm 0.08$ & $0.19 \pm 0.08$ & 0.001 \\
\hline
\end{tabular}

e-GFR: estimate Glomerular Filtration Rate; HOMA-R: Homeostasis Model Assessment Insulin Resistance; AUC-IRI: Area Under the Curve of Immune Reactive Insulin; LDL: Low Density Lipoprotein; HDL: High Density Lipoprotein; RLP: Remnant Like Particle; PAI-1: Plasminogen Activator Inhibitor-1

Table 4: Comparison changes in blood biochemistry and flow-mediated dilatation.

\begin{tabular}{|c|c|c|c|c|}
\hline \multirow{3}{*}{ Variable } & \multicolumn{2}{|c|}{ no change FMD group; $n=25$} & \multicolumn{2}{|c|}{ improved FMD group; $\mathbf{n}=25$} \\
\hline & \multicolumn{2}{|c|}{$(\Delta \%$ FMD of $\leq 0.7 \%)$} & \multicolumn{2}{|c|}{$\llbracket \Delta \%$ FMD of $>0.7 \% \rrbracket$} \\
\hline & Before & After 6 months & Before & After 6 months \\
\hline Weight (kg) & $68.5 \pm 9.7$ & $65.2 \pm 9.2^{*}$ & $68.7 \pm 10.2$ & $65.4 \pm 10.2^{*}$ \\
\hline Body mass index $\left(\mathrm{kg} / \mathrm{m}^{2}\right)$ & $24.9 \pm 2.4$ & $23.7 \pm 2.5^{*}$ & $24.9 \pm 3.6$ & $23.7 \pm 3.7^{*}$ \\
\hline Body fat quantity $(\mathrm{kg})$ & $18.0 \pm 4.3$ & $15.7 \pm 4.2^{*}$ & $18.6 \pm 5.9$ & $16.2 \pm 6.3^{*}$ \\
\hline Body fat percentage (\%) & $26.6 \pm 5.6$ & $24.3 \pm 5.8^{* *}$ & $27.3 \pm 6.0$ & $24.9 \pm 7.0^{*}$ \\
\hline Muscle quantity $(\mathrm{kg})$ & $47.5 \pm 8.2$ & $46.5 \pm 7.8^{* * *}$ & $46.2 \pm 6.0$ & $45.1 \pm 6.0^{* *}$ \\
\hline Muscle percentage (\%) & $69.4 \pm 5.3$ & $71.5 \pm 5.6^{*}$ & $68.7 \pm 5.7$ & $70.9 \pm 6.7^{*}$ \\
\hline Water quantity $(\mathrm{kg})$ & $37.0 \pm 6.4$ & $36.3 \pm 6.0^{* *}$ & $36.0 \pm 4.6$ & $35.2 \pm 4.6^{* *}$ \\
\hline Extracellular water & 0.38 & 0.38 & 0.38 & 0.39 \\
\hline Total body water & $(0.38,0.39)$ & $(0.38,0.39)$ & $(0.38,0.39)$ & $(0.38,0.39)$ \\
\hline Systolic blood pressure $(\mathrm{mmHg})$ & $130.1 \pm 13.0$ & $127.1 \pm 11.7$ & $136.9 \pm 16.7$ & $134.1 \pm 15.9$ \\
\hline Diastolic blood pressure $(\mathrm{mmHg})$ & $80.1 \pm 10.7$ & $78.2 \pm 9.8$ & $80.1 \pm 10.1$ & $78.6 \pm 9.3^{* * *}$ \\
\hline White blood cell $\left(\times 10^{2} / \mu \mathrm{L}\right)$ & $60.2 \pm 15.0$ & $55.8 \pm 12.9$ & $63.0 \pm 14.4$ & $65.7 \pm 16.2$ \\
\hline Red blood cell $(\times 104 / \mu \mathrm{L})$ & $472.7 \pm 42.1$ & $491.0 \pm 43.1^{*}$ & $479.6 \pm 31.2$ & $505.0 \pm 32.6^{*}$ \\
\hline Hemoglobin $(\mathrm{g} / \mathrm{dL})$ & $14.4 \pm 1.0$ & $14.9 \pm 1.0^{* *}$ & $14.5 \pm 1.3$ & $15.1 \pm 1.4^{* *}$ \\
\hline Hematocrit (\%) & $43.0 \pm 2.6$ & $44.5 \pm 2.4^{* *}$ & $43.6 \pm 3.2$ & $45.1 \pm 3.5^{* * *}$ \\
\hline Platelet $\left(\times 10^{4} / \mu \mathrm{L}\right)$ & $21.0 \pm 4.0$ & $19.7 \pm 3.9^{* * *}$ & $22.3 \pm 5.2$ & $21.3 \pm 5.0^{* * *}$ \\
\hline Plasma creatinine $(\mathrm{mg} / \mathrm{dL})$ & $0.79 \pm 0.12$ & $0.81 \pm 0.16$ & $0.84 \pm 0.16$ & $0.87 \pm 0.17$ \\
\hline $\mathrm{e}-\mathrm{GFR}(\mathrm{mg} / \mathrm{dL})$ & $75.1 \pm 15.4$ & $73.1 \pm 13.4$ & $71.6 \pm 13.9$ & $69.4 \pm 14.5$ \\
\hline Urine albumin $(\mathrm{mg} / \mathrm{dL})$ & $13.8(6.2,62.8)$ & $10.8(6.0,23.6)$ & $18.0(10.1,46.6)$ & $12.4(8.6,26.9)$ \\
\hline Uric acid $(\mathrm{mg} / \mathrm{dL})$ & $5.3 \pm 1.4$ & $4.5 \pm 1.0^{* *}$ & $5.9 \pm 1.2$ & $4.8 \pm 1.2^{* *}$ \\
\hline HemoglobinA1c (\%) & $7.2 \pm 0.5$ & $6.8 \pm 0.4^{*}$ & $6.8 \pm 0.3 \dagger$ & $6.4 \pm 0.4^{* *}$ \\
\hline 1,5-anhydroglucitol $(\mu \mathrm{g} / \mathrm{dL})$ & $10.9 \pm 6.2$ & $2.5 \pm 2.7^{*}$ & $11.3 \pm 5.4$ & $1.8 \pm 0.7^{*}$ \\
\hline
\end{tabular}

Plasma glucose level (mg/dL) 


\begin{tabular}{|c|c|c|c|c|}
\hline Fasting & $130.1 \pm 16.6$ & $114.0 \pm 12.7^{*}$ & $128.2 \pm 15.8$ & $115.6 \pm 23.4^{*}$ \\
\hline After 1 hour & $242.7 \pm 34.1$ & $212.8 \pm 28.3^{*}$ & $234.7 \pm 32.9$ & $221.6 \pm 38.4$ \\
\hline After 2 hours & $238.7 \pm 51.0$ & $204.8 \pm 45.6^{* *}$ & $233.0 \pm 42.2$ & $222.7 \pm 55.4$ \\
\hline Incremental glucose peak (mg/dL) & $122.6 \pm 31.4$ & $107.2 \pm 31.0^{* * *}$ & $121.8 \pm 31.4$ & $121.4 \pm 36.7$ \\
\hline \multicolumn{5}{|l|}{ Immune reactive insulin $(\mu \mathrm{U} / \mathrm{mL})$} \\
\hline Fasting & $6.3(4.1,9.1)$ & $5.4(3.5,8.5)$ & $7.9(4.8,9.9)$ & $4.4(3.0,8.7)^{\star}$ \\
\hline After 1 hour & $40.7(22.9,54.3)$ & $37.6(18.1,58.9)$ & $41.1(25.7,69.3)$ & $27.0(19.9,60.4)^{* *}$ \\
\hline After 2 hours & $47.9(27.4,65.9)$ & $42.8(22.6,60.0)$ & $50.7(32.1,93.5)$ & $43.2(27.0,70.3)^{* *}$ \\
\hline HOMA-R & $2.4(1.4,3.1)$ & $1.7(0.9,2.3)^{* * *}$ & $2.6(1.5,3.2)$ & $1.3(0.8,2.7)^{\star *}$ \\
\hline AUC-IRI $(\mu \mathrm{U} / \mathrm{mL})$ & $67.3(36.3,94.8)$ & $64.2(30.3,92.0)$ & $70.3(43.2,119.1)$ & $51.8(35.4,93.3)^{* *}$ \\
\hline \multicolumn{5}{|l|}{ C peptide $(\mathrm{ng} / \mathrm{mL})$} \\
\hline Fasting & $2.1(1.5,2.4)$ & $1.7(1.4,2.2)^{* * *}$ & $2.2(1.9,2.7)$ & $1.7(1.3,2.3)^{* *}$ \\
\hline After 1 hour & $5.2(4.5,7.0)$ & $5.0(3.4,6.9)$ & $5.6(4.3,8.6)$ & $4.4(3.7,8.4)^{* * *}$ \\
\hline After 2 hours & $8.2(6.2,10.0)$ & $7.8(5.8,10.7)$ & $10.0(6.5,14.3)$ & $8.0(7.0,11.4)^{* * *}$ \\
\hline Total cholesterol (mg/dL) & $157.6 \pm 21.0$ & $161.4 \pm 22.6$ & $172.2 \pm 28.1$ & $165.8 \pm 38.8$ \\
\hline LDL cholesterol (mg/dL) & $88.1 \pm 18.7$ & $89.4 \pm 17.8$ & $93.1 \pm 27.7$ & $92.2 \pm 29.4$ \\
\hline HDL cholesterol (mg/dL) & $48.4 \pm 8.8$ & $50.9 \pm 9.8$ & $53.8 \pm 26.4$ & $51.2 \pm 9.2$ \\
\hline \multicolumn{5}{|l|}{ Plasma triglyceride $(\mathrm{mg} / \mathrm{dL})$} \\
\hline Fasting & $99.0 \pm 33.7$ & $96.2 \pm 33.3$ & $140.8 \pm 64.0 \dagger$ & $95.1 \pm 34.4^{*}$ \\
\hline After 1 hour & $119.8 \pm 32.9$ & $124.2 \pm 43.7$ & $165.2 \pm 60.7 \dagger$ & $121.0 \pm 38.9^{*}$ \\
\hline After 2 hours & $155.6 \pm 49.5$ & $154.4 \pm 57.9$ & $212.9 \pm 76.9 \dagger$ & $152.5 \pm 48.3^{*}$ \\
\hline \multirow[t]{2}{*}{ AUC-triglyceride (mg/dL) } & 248 & 251.5 & 329 & 250 \\
\hline & $(193.9,290.6)$ & $(197.5,281.6)$ & $(246.0,425.1) \dagger$ & $(176.8,310.1)^{*}$ \\
\hline RLP cholesterol (mg/dL) & $4.1(2.6,6.0)$ & $4.1(2.6,5.1)$ & $6.1(4.2,9.8) \dagger$ & $3.7(2.4,4.7)^{*}$ \\
\hline $\mathrm{C}$ reactive protein $(\mathrm{mg} / \mathrm{dL})$ & $0.07(0.03,0.18)$ & $0.06(0.03,0.11)$ & $0.12(0.04,0.29)$ & $0.10(0.04,0.20)^{* * *}$ \\
\hline Brain natriuretic peptide $(\mathrm{pg} / \mathrm{mL})$ & $31.4(14.0,59.2)$ & $19.4(9.1,39.7)$ & $28.8(14.2,59.0)$ & $22.0(10.8,58.0)$ \\
\hline PAI-1 (ng/mL) & $16.9 \pm 6.5$ & $12.9 \pm 4.8^{* *}$ & $20.3 \pm 8.6$ & $14.7 \pm 5.1^{*}$ \\
\hline Total ketone body $(\mu \mathrm{mol} / \mathrm{L})$ & $95.0(65.5,143.0)$ & $134.0(86.0,152.5)$ & $80.0(50.0,138.5)$ & $191.0(107.0,336.0)^{*}$ \\
\hline \%flow-mediated dilatation (\%) & $5.5(3.4,6.0)$ & $4.7(2.8,5.8)$ & $2.6(1.9,3.6) \dagger$ & $4.6(3.8,5.1)^{\star}$ \\
\hline Absolute change in diameter $(\mathrm{mm})$ & $0.19(0.15,0.26)$ & $0.18(0.12,0.24)^{* *}$ & $0.11(0.09,0.16) \dagger$ & $0.19(0.16,0.24)^{*}$ \\
\hline \multicolumn{5}{|c|}{$\begin{array}{l}{ }^{*} \mathrm{p}<0.0001 \text { vs. baseline, }{ }^{* *} \mathrm{p}<0.001 \text { vs. baseline, }{ }^{* * *} \mathrm{p}<0.05 \text { vs. baseline, } \dagger \mathrm{p}<0.05 \text { vs. baseline of no change in FMD group, FMD: Flow- } \\
\text { Mediated Dilatation, e-GFR: estimate Glomerular Filtration Rate, HOMA-R: Homeostasis Model Assessment Insulin Resistance, AUC- } \\
\text { IRI: Area Under the Curve of Immune Reactive Insulin, LDL: Low Density Lipoprotein, HDL: High Density Lipoprotein, RLP: Remnant } \\
\text { Like Particle, PAI-1: Plasminogen Activator Inhibitor-1. }\end{array}$} \\
\hline
\end{tabular}

hematocrit while platelet count decreased slightly. Plasma uric acid and urinary albumin level decreased significantly while serum creatinine level was slightly increased. No significant change was observed in BNP.

The \%FMD, the primary endpoint of this study, improved significantly (median; $3.5 \%$ to $4.7 \%$, p $<0.0001$ ) while CRP and PAI-1 decreased significantly. Absolute change in brachial artery diameter was also increased significantly after 6 months of treatment.

\section{Comparing between the median values of changes in $\%$ FMD}

We divided the patients into two groups according to the median values of the changes in \%FMD (no change FMD group $[\triangle \% \mathrm{FMD}$ of $\leq 0.7 \%$ ], $n=25$; and improved FMD group [ $\triangle \%$ FMD of $>0.7 \%$ ], $\mathrm{n}=25$ patients).
As shown in Table 4, we noted significant differences in $\mathrm{HbA}_{1 \mathrm{C}}$, fasting and postprandial TG and RLP-C between the two groups. We also noted that the baseline \%FMD in the improved FMD group was significantly lower than that in the no change FMD group.

After 6 months of treatment, both groups exhibited similar changes in anthropometric measurements and hemodynamic data. Although both groups showed significant reduction in $\mathrm{HbA}_{1 \mathrm{C}}$, fasting $P G$, and HOMA-R, fasting and postprandial insulin secretion decreased significantly in the improved FMD group.

Among the lipid profiles, fasting and postprandial TG (e.g. AUCTG and RLP-C) were also significantly decreased, in the improved FMD group only.

Furthermore decreased CRP level and significant increment in total ketone body occurred only in the improved FMD group. 
Table 5: Regression analysis for predicting \%flow-mediated dilatation improvement.

\begin{tabular}{|c|c|c|c|c|}
\hline \multirow{2}{*}{ variable } & \multicolumn{2}{|c|}{ univariate } & \multicolumn{2}{|c|}{ multivariate } \\
\hline & $\mathrm{t}$ & p-value & $\mathrm{t}$ & p-value \\
\hline Age & -0.119 & 0.91 & -0.065 & 0.95 \\
\hline Sex & 0.892 & 0.38 & 1.239 & 0.22 \\
\hline Changes in weight & -0.154 & 0.88 & & \\
\hline Changes in body fat percentage & 0.593 & 0.56 & & \\
\hline Changes in body muscle percentage & 0.919 & 0.36 & & \\
\hline Changes in water quantity & 0.918 & 0.36 & & \\
\hline Changes in hematocrit & -0.036 & 0.97 & & \\
\hline Changes in urine albumin & 1.727 & 0.09 & & \\
\hline Changes in e-GFR & 0.125 & 0.9 & & \\
\hline Changes in uric acid & -0.074 & 0.94 & & \\
\hline Changes in hemoglobin A1c & -2.438 & 0.02 & -1.708 & 0.09 \\
\hline Changes in 1,5-anhydro-glucitol & -0.615 & 0.54 & & \\
\hline Changes in fasting plasma glucose & -0.214 & 0.83 & & \\
\hline Changes in incremental glucose peak & 0.423 & 0.67 & & \\
\hline Changes in fasting IRI & -2.433 & 0.02 & & \\
\hline Changes in AUC-IRI & -2.312 & 0.02 & -0.775 & 0.44 \\
\hline Changes in HOMA-R & -1.769 & 0.08 & & \\
\hline Changes in LDL cholesterol & -1.025 & 0.31 & & \\
\hline Changes in HDL cholesterol & -0.107 & 0.92 & & \\
\hline Changes in fasting triglyceride & -5.939 & $<0.0001$ & -5.24 & $<0.0001$ \\
\hline Changing in AUC-triglyceride & -6.988 & $<0.0001$ & -5.94 & $<0.0001$ \\
\hline Changes in RLP cholesterol & -4.971 & $<0.0001$ & & \\
\hline Changes in C-reactive protein & 0.299 & 0.77 & & \\
\hline Changes in BNP & 1.618 & 0.11 & & \\
\hline Changes in PAI-1 & -0.428 & 0.67 & & \\
\hline Changing total ketone body & 2.057 & 0.04 & 1.155 & 0.25 \\
\hline
\end{tabular}

e-GFR: estimate Glomerular Filtration Rate, HOMA-R: Homeostasis Model Assessment Insulin Resistance, AUC-IRI: Area Under the Curve of Immune Reactive Insulin, LDL: Low Density Lipoprotein, HDL: High Density Lipoprotein, RLP: Remnant Like Particle, BNP: Brain Natriuretic Peptide, PAI-1: Plasminogen Activator Inhibitor-1

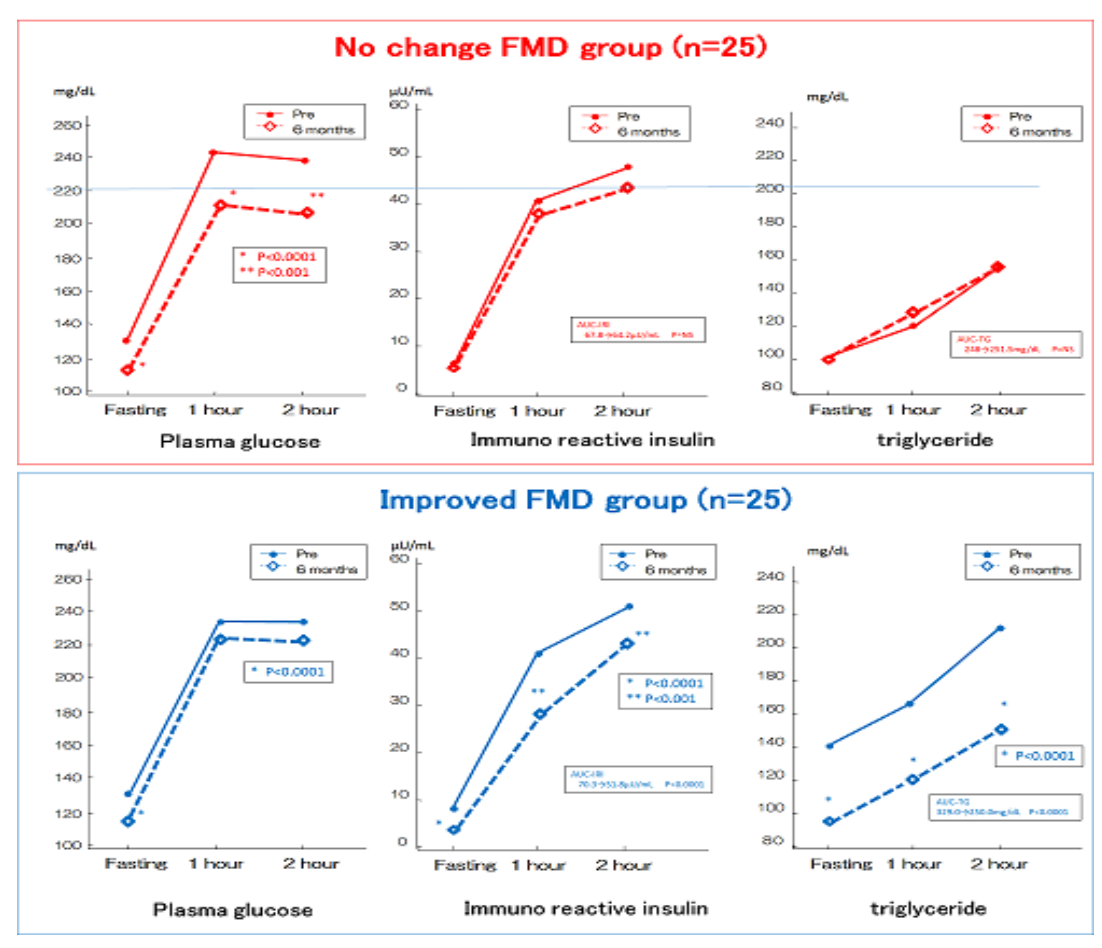

Figure 1: Plasma glucose, immune reactive insulin, and triglyceride levels during the cookie meal test at baseline and after 6 months. * indicates $p<0.0001$ vs. baseline. ${ }^{* *}$ indicates $\mathrm{p}<0.001$ vs. baseline. Comparisons of data before and after treatment were performed using the paired t-test. 


\section{Regression analysis of factors predicting \%FMD improvement}

Univariate and multivariate regression analysis results are shown in Table 5. Univariate analysis revealed that baseline \%FMD, baseline absolute change in the diameters of the brachial artery, changes in $\mathrm{HbA}_{1 \mathrm{C}}$ level, fasting and postprandial insulin and TG levels, and plasma total ketone body level were associated with the changes in \%FMD. Multiple regression analysis revealed that the strongest independent factor predicting FMD changes was change in AUCTG (Figure 1).

\section{DISCUSSION}

In this study, we noted that 6 months of treatment with empagliflozin improved not only the glycemic control but also multiple coronary risk including the blood pressure, obesity, insulin resistance, hypertriglyceridemia, and hyperuricemia. In addition, it ameliorated several atherosclerotic markers, such as inflammation, thrombogenicity, and endothelial function. Among these factors, improvement in both fasting and postprandial hypertriglyceridemia was the strongest independent factor predicting improved of endothelial dysfunction in diabetic patients with CAD, treated with empaglifolozin.

Type $2 \mathrm{DM}$ is a major risk factor for the progression of atherosclerosis and development of cardiovascular disease. Furthermore, type 2 DM patients experience substantial morbidity and mortality from cardiovascular disease [1,2]. Most observational studies that have reported an association between glucose control and cardiovascular disease and glycemic control have indicated that the association may play an important role in the prevention of CAD, among patients with diabetes [22]. However, the fact that lowering of glucose reduces the rate of cardiovascular events and death has not been convincingly shown [23]. Rather, there is concern that intensive glucose control may be associated with adverse cardiovascular outcomes [24,25]. Among various oral glucose lowering agents with different mechanisms of action, SGLT2 inhibitors are one of the few oral diabetic drugs that achieved significant reduction in major cardiovascular outcomes in large randomized controlled trials [4,5]. Along with their glucose lowering effect, SGLT2 inhibitors possess multidimensional properties that may positively affect several recognized cardiovascular risk factors. SGLT2 inhibitors exert a glucose lowering effect by enhancing glucosuria, independently of insulin secretion [26]. They then indirectly improve insulin sensitivity by reducing glucose toxicity [27]. Furthermore, weight reduction results from calorie loss because of glucosuria and lipid breakdown [28]. Blood pressure is commonly decreased because of SGLT2 inhibitors' diuretic effects without sympathetic nerve stimulation [29]. A reduction in serum uric acid level is attributed to enhanced urinary excretion [30]. An increase in hematocrit is presumed to involve enhanced erythropoiesis, in addition to hemoconcentration, which may also exert positive effects [31]. We also recognized all these favorable changes following SGLT2 inhibitors administration. As shown in Steno-2 trial, it has considered that intensive intervention with multiple drug combinations and behavior modification, had sustained beneficial effects with respect to vascular complications and rate of cardiovascular deaths [32]. We, therefore, believe that all these favorable effects of SGLT2 inhibitors may be linked to the improvement in endothelial function. This finally contributes to reduced cardiovascular events accompanied by DM; therefore
SGLT2 inhibitors should be a possible anti-diabetic agent especially for diabetic patients complicated by cardiovascular disease.

The present study also revealed multiple favorable effects similar to that reported in previous studies [27-32]. However, the precise mechanisms underlying the cardiovascular benefits of SGLT2 inhibitors remain to be elucidated. Recently, Inzucchi et al. demonstrated that changes in markers such as plasma volume, hematocrit, and hemoglobin, were the most important mediators of lower cardiovascular event rate [31]. Indeed, in the EMPA-REG OUTCOME and CANVAS trials, the greatest benefit of SGLT2 inhibitors was the reduction in hospitalization for heart failure $[4,5]$. Our data also revealed a significantly decreased water quantity and significantly increased red blood cell count, hemoglobin, and hematocrit. Thus, the mitigating effect of SGLT2 inhibitors on volume overload probably contributed to the reduction in heart failure risk and might be one of the potential mechanisms for the reduction in cardiovascular events with SGLT2 inhibitors. However, the Hisayama study showed that both elevated and decreased hematocrit were associated with increased cardiovascular disease [33]. Whereas, in our study, we observed that the hematocrit and hemoglobin levels in the FMD improved group, were similar to those in the no change FMD group. Furthermore, BNP level indicated no changes in both groups. Therefore, we could not conclude that the only volume reduction effect of SGLT2 inhibitors was the most plausible mechanism of cardiovascular benefit of SGLT2 inhibitors.

On the other hand, significant reductions in both fasting and postprandial triglyceride and insulin were observed only in the improved FMD group, and the improvement of insulin resistance in the improved FMD group was greater than that in the no change FMD group. In addition, multivariate regression analysis revealed that the improvement in fasting and postprandial hypertriglyceridemia was the strongest independent factor predicting improvement in endothelial dysfunction in diabetic patients with CAD. In general, type 2 DM commonly demonstrates elevated TG, low HDL-C, and the predominance of small dense LDL particles due to insulin resistance and this type of dyslipidemia is considered to be atherogenic dyslipidemia [34,35]. Sone et al. demonstrated that the serum triglyceride level was a leading predictor of CAD, which is comparable to LDL-C in Japanese patients with type 2 DM [36]. Furthermore, the cardiovascular risk associated with this atherogenic dyslipidemia is uncorrelated with, and additive to LDL-C levels. Researchers are currently focusing on atherogenic dyslipidemia as a residual risk factor for cardiovascular events in statin-treated diabetic patients [35]. Therefore, we speculate that anti-atherosclerotic effects of SGLT2 inhibitors, such as reduction in hypertriglyceridemia and improved insulin resistance is important, and should play a major role in the reduction of cardiovascular events. Of note, we previously demonstrated that I-glucosidase inhibitor improved atherogenic dyslipidemia and insulin resistance. These effects are associated with the beneficial impact of I-glucosidase inhibitors on oxidative stress and improvement in endothelial function in diabetic patients with CAD [37]. We consider I-glucosidase inhibitors to be a comparable anti-diabetic agent with SGLT2 inhibitors in that they both have ameliorating effects on hyperglycemia without the induction of insulin secretion. Similar to EMPA-REG OUTCOME and CANVAS programs, the first prospective randomized intervention trial on t-glucosidase inhibitors, the Study to Prevent Non-insulin-Dependent Diabetes Mellitus (STOP-NIDDM) trial, demonstrated that treatment with 
an I-glucosidase inhibitors, acarbose, is associated with a significant reduction in the risk of cardiovascular disease [38]. Based on these observations, we are of the opinion that one of the beneficial effects of empagliflozin on endothelial dysfunction may be associated with the improvement in hypertriglyceridemia and insulin resistance.

The role of insulin in regulating glucose disposal in skeletal muscle is well understood [39]. In skeletal muscle, insulin enhanced vasodilatation and capillary recruitment, consequently increasing a blood flow, and also promotes the rate of glucose disposal [40]. Therefore, skeletal muscle blood flow is important for insulinstimulated glucose output. In the state of insulin resistance, vasoactive actions of insulin in the skeletal muscle is blunted due to an unresponsive nitric oxide synthase and consequently leading the skeletal muscle blood flow reductions and then occurred hyperglycemia [41]. Thus, impaired insulin-dependent rise in skeletal muscle blood flow can be attributed to the diabetic milieu, and this could contribute to the progression to type $2 \mathrm{DM}$ as well as to the development of atherosclerosis [42]. In this study, we recognized not only the improvement of hyperglycemia but also of insulin resistance after SGLT2 inhibitors treatment. And this improvement was accompanied by \%FMD improvement which is indirectly indicated skeletal muscle blood flow increment. From this standpoint, we considered that SGLT2 inhibitors are useful anti-diabetic agents for the diabetic patients who were highly associated with cardiovascular disease.

Intriguingly, though the strongest independent factor of \%FMD improvement was the reduction in postprandial TG levels, regression analysis also revealed that total ketone body increment was associated with the improvement in endothelial function. Some researchers suggested that under a mild hyperketonemia condition caused by SGLT2 inhibitors, the heart preferentially metabolizes ketone bodies instead of glucose, as an alternative fuel source, thereby improving myocardial work efficiency and function [43]. In fact, Aubert et al. indicated that the hypertrophied and failing myocardium shifts to ketone body as a significant fuel source for oxidative adenosine triphosphate production in mouse models [44]. Our data also support this hypothesis; however, a further large size, randomized controlled study is warranted.

\section{LIMITATIONS}

Several limitations in the present study warrant consideration. Firstly, this was a study with a single-arm design and no control group. Therefore, changes that might be observed without SGLT2 inhibitors treatment could not be compared in this study. However, in our previous other study, we observed not so significant change in \%FMD among patients who underwent just dietary and exercise therapy for 6 months [19]. Thus, we believed that SGLT2 therapy may have an additive beneficial influence on endothelial dysfunction in comparison with none SGLT2 therapy. Secondly, although we calculated the required sample size according to a previous study, the number of participants required to detect a possible association between changes in FMD and those of several biochemical markers was relatively small. Thirdly, although participants were prohibited from changing the dose of concomitant drugs or adding any other drugs throughout the study, several concomitant drugs (such as statin, angiotensin converting enzyme inhibitor, angiotensin receptor blocker) were used, which had the potency of improving endothelial function; therefore some bias was likely to have occurred in our results.

\section{CONCLUSIONS}

In conclusion, empagliflozin improved not only glycemic control but also insulin resistance and hypertriglyceridemia. These ameliorations of metabolic abnormality were strongly associated with improvements in concomitant endothelial dysfunction among diabetic patients with CAD. Although the mechanism of the cardiovascular benefit of SGLT2 inhibitors might be multifactorial, reduction in the plasma triglyceride may be mostly related to the cardiovascular benefit of SGLT2 inhibitors.

\section{ACKNOWLEDGEMENTS}

None

\section{DISCLOSURE}

There is no conflict of interest in this study.

\section{REFERENCES}

1. Mak KH, Moliterno DJ, Granger CB, Miller DP, White HD, Wilcox $\mathrm{RG}$, et al. Influence of diabetes mellitus on clinical outcome in the thrombolytic era of acute myocardial infarction, GUSTO- I Investigators. Global Utilization of Streptokinase and Tissue Plasminogen Actibator for Occluded Coronary Arteries. J Am Coll Cardiol. 1997;30:171-179.

2. Malmberg K, Yusuf S, Gerstein H, Brown J, Zhao F, Hunt D, et al. Impact of diabetes on long-term prognosis in patients with unstable angina and non-Q wave myocardial infarction: results of the OASIS (Organization to Assess Strategies for Ischemic Syndrome) Registry. Circulation. 2000;102:1014-1019.

3. Vallon V. The mechanisms and therapeutic potential of SGLT2 inhibitors in diabetes mellitus. Annu Rev Med. 2015;66:255-270.

4. Zinman B, Wanner C, Lachin JM, Fitchett D, Bluhmki E, Hantel S, et al. Empagliflozin, cardioavascular outcomes, and mortality in type 2 diabetes. N Engl J Med. 2015;373:2117-2128.

5. Mahaffey KW, Neal B, Perkovic V, de Zeeuw D, Fulcher G, Erondu $\mathrm{N}$, et al. Canagliflozin for primary and secondary prevention of cardiovascular events: Results from the CANVAS Program (Canagliflozi Cardiovascular Assessment Study). Circulation. 2018;137:323-334.

6. Heerspink HJ, Perkins BA, Fitchett DH, Husain M, Cherney DZ. Sodium glucose cotransporter 2 inhibitors in the treatment of diabetes mellitus: cardiovascular and kidney effects, potential mechanisms, and clinical applications. Circulation. 2016;134:752-772.

7. Nauck MA, Del Prato S, Meier JJ, Duran-Garcia S, Rohwedder K, Elze $\mathrm{M}$, et al. Dapagliflozin versus glipzide as add-on therapy in patients with type 2 diabetes who have inadequate glycemic control with metformin: a randomized, 52-week, double-blind, active-controlled noninferiority trial. Diabetes Care. 2011;34:2015-2022.

8. Sattar N, McLaren J, Kristensen SL, Preiss D, McMurray JJ. SGLT2 inhibition and cardiovascular events: Why did EMPA-REG Outcomes surprise and what were the likely mechanisms? Diabetologia. 2016;59:1333-1339.

9. Anderson TJ, Uehata A, Gerhard MD, Meredith IT, Knab S, Delagrange $\mathrm{D}$, et al. Close relation of endothelial function in the human coronary and peripheral circulation. J Am Coll Cardiol. 1995;26:1235-1241.

10.Drexler H. Endothelium as a therapeutic target in heart failure. Circulation. 1998;98:2652-2655.

11. Celermajer DS, Sorensen KE, Gooch VM, Spiegelhalter DJ, Miller OI, Sullivan ID, et al. Non-invasive detection of endothelial dysfunction in 
children and adults at risk of atherosclerosis. Lancet. 1992;340:11111115 .

12. Neuteufl T, Heher S, Katzenschlager R, Wolfl G, Kostner K, Maurer G, et al. Late prognostic value of flow-mediated dilatation in the brachial artery of patients with chest pain. Am J Cardiol. 2000;86:207-210.

13. Sawada T, Emoto T, Motoji Y, Hashimoto M, Kageyama H, Terashita $\mathrm{D}$, et al. Possible association between non-invasive parameter of flowmediated dilatation in brachial artery and whole coronary plaque vulnerability in patients with coronary artery disease. Int J Cardiol. 2013;166:613-620.

14. Neunteufl T, Katzenchlager R, Hassan A, Klaar U, Shwarzacher S, Glogar D, et al. Systemic endothelial dysfunction is related to the extent and severity of coronary artery disease. Atherosclerosis. 1997;129:111118 .

15.Tousoulis D, Oikonomou E, Siasos G, Christine C, Zaromitidou M, Kioufis S, et al. Dose-dependent effects of short term atorvastatin treatment on arterial wall properties and on indices of left ventricular remodeling in ischemic heart failure. Atherosclerosis. 2013;227:367372 .

16. Cha K, Chertow GM, Gonzalez J, Lazarus JM, Wilmore DW. Multifrequency bioelectrical impedance estimates the distribution of body water. J Appl Physiol. 1995;79:1316-1319.

17. Kurinami N, Sugiyama S, Nishimura H, Morita A, Yoshida A, Hieshima $\mathrm{K}$, et al. Clinical factors associated with initial decrease in body-fat percentage induced by add-on sodium-glucose co-transporter 2 inhibitors in patient with type 2 diabetes mellitus. Clin Drug Investig. 2018;38:19-27.

18. Harano Y, Miyawaki T, Nabiki J, Shibachi M, Adachi T, Ikeda M, et al. Development of cookie test for the simultaneous determination of glucose intolerance, hyperinsulinemia, insulin resistance and postprandial dyslipidemia. Endocr J. 2006;53:173-180.

19.Sawada T, Tsubata H, Hashimoto N, Takabe M, Miyata T, Aoki $\mathrm{K}$, et al. Effects of 6-month eicosapentaenoic acid on postprandial hypertriglycemia, hyperlipidemia, insulin secretion ability, and concomitant endothelial dysfunction among newly-diagnosed impaired glucose metabolism patients with coronary artery disease. An open label, single blinded, prospective randomized controlled trial. Cardiovasc Diabetol. 2016;15:121.

20.Emoto T, Sawada T, Hashimoto M, Kageyama H, Terashita D, Mizoguchi T, et al. Effect of 3-month repeated administration of miglitol on vascular endothelial function in patients with diabetes mellitus and coronary artery disease. Am J Cardiol. 2012;109:42-46.

21. Shigiyama F, Kumashiro N, Miyagi M, Ikehara K, Kanda E, Uchino H, et al. Effectiveness of dapagliflozin on vascular endothelial function and glycemic control in patients with early-stage type 2 diabetes mellitus: DEFENCE study. Cardiovasc Diabetol. 2017;16:84.

22.Selvin E, Marinopoulos S, Berkenblit G, Rami T, Brancati FL, Powe NR, et al. Meta-analysis: Glycosylated hemoglobin and cardiovascular disease in diabetes mellitus. Ann Intern Med. 2004;141:421-431.

23. Green JB, Bethel MA, Armstrong PW, Buse JB, Engel SS, Garg J, et al. Effect of sitagliptine on cardiovascular outcomes in type 2 diabetes. $\mathrm{N}$ Engl J Med. 2015;373:232-242.

24.Udell JA, Cavender MA, Bhatt DL, Chatterjee S, Farkouh ME, Scirica BM. Glucose-lowering drugs or strategies and cardiovascular outcomes in patients with or at risk for type 2 diabetes: a meta-analysis of randomised controlled trials. Lancet Diabetes Endocrinol. 2015;3:356366.

25.ACCORD Study Group, Gerstein HC, Miller ME, Genuth S, IsmailBeigi F, Buse JB, et al. Long-term effects of intensive glucose lowering on cardiovascular outcomes. N Engl J Med. 2011;364:818-828.
26.Vallon V, Thomsn SC. Targeting renal glucose reabsorption to treat hyperglycaemia: the pleiotropic effects of SGLT2 inhibition. Diabetologia. 2017;60:215-225.

27. Abdul-Ghani MA, Norton L, Defrozo RA. Role of sodium-glucose cotransporter type 2 (SGLT2) inhibitors for the treatment of type 2 diabetes. Endocr Rev. 2011;32:515-531.

28. Cai X, Ji L, Chen Y, Yang W, Zhou L, Han X, et al. Comparisons of weight changes between sodium-glucose cotranspoter 2 inhibitors treatment and glucagon-like peptide- 1 analogs treatment in type 2 diabetes patients: a meta-analysis. J Diabetes Investig. 2017;8:510-517.

29.Mazidi M, Rezaie P, Gao HK, Kengne AP. Effect of sodium-glucose cotransport-2 inhibitors on blood pressure in people with type 2 diabetes mellitus: A systematic review and meta-analysis of 43 randomized control trials with 225228 patients. J Am Heart Assoc. 2017;6: e005686.

30.Zhao Y, Xu L, Tian D, Xia P, Zheng H, Wang L, et al. Effects of sodiumglucose cotransporter 2 (SGLT2) inhibitors on serum uric acid level: a meta-analysis of randomized controlled trials. Diabetes Obes Metab. 2018;20:458-462.

31. Inzucchi SE, Zinman B, Fitchett D, Wanner C, Ferrannini E, Schumacher M, et al. How does empagliflozin reduce cardiovascular mortality? Insights from a medication analysis of the EMPA-REG OUTCOME Trial. Diabetes Care. 2018;41:356-363.

32.Gaede P, Lund-Andersen H, Parving HH, Pedersen O. Effect of a multifactorial intervention on mortality in type 2 diabetes. $\mathrm{N}$ Engl J Med. 2008;358:580-591.

33.Gotoh S, Hata J, Ninomiya T, Hirakawa Y, Nagata M, Mukai N, et al. Hematocrit and the risk of cardioivascular disease in a Japanese community: The Hisayama Study. Atherosclerosis. 2015;242:199-204.

34.Fruchart JC, Sacks F, Hermans MP, Assmann G, Brown WV, Ceska R, et al. The Residual Risk Reduction Initiative: a call to action to reduce residual vascular risk in patients with dyslipidemia. Am J Cardiol. 2008; 102:1K-34K.

35.Wu L, Parhofer KG. Diabetic dyslipidemia. Metabolism. 2014;63:14691479.

36.Sone H, Tanaka S, Tanaka S, Iimuro S, Oida K, Yamasaki Y, et al. Serum level of triglycerides is a potent risk factor comparable to LDL cholesterol for coronary heart disease in Japanese patients with type 2 diabetes: subanalysis of the Japan Diabetes Complication Study (JDCS). J Clin Endocrinol Metab. 2011;96:3448-3456.

37. Sawada T, Shiotani H, Terashita D, Nagasawa Y, Kim SS, Koide M, et al. Comparison of effects of alpha-glucosidase inhibitors and glinide drugs on endothelial dysfunction in diabetic patients with coronary artery disease. Circ J. 2014;78:248-255.

38. Chiasson JL, Josse RG, Gomis R, Hanefeld M, Karasik A, Laakso M, et al. STOP-NIDDM Trial Group. Acarbose treatment and the risk of cardiovascular disease and hypertension in patients with impaired glucose tolerance: The STOP-NIDDM trial. JAMA. 2003;290:486-494.

39.Baron AD, Steinberg H, Brechtel G, Johnson A. Skeltal muscle blood flow independently modulates insulin-mediated glucose uptake. Am J Physiol. 1994;266:E248-E253.

40.Newman JM, Ross RM, Richards SM, Clark MG, Rattigan S. Insulin and contraction increase nutritive blood flow in rat muscle in vivo determined by microdialysis of L-(14C) glucose. J Physiol. 2007;585:217229.

41. Mather KJ, Steinberg HO, Baron AD. Insulin resistance in the vasculature. J Clin Invest. 2013;123:1003-1004.

42.Laakso M, Edelman SV, Brechtel G, Baron AD. Impaired insulin mediated skeltal muscle blood flow in patients with NIDDM. Diabetes. 1992;41:1076-1083. 
43. Mudalier S, Alloju S, Henry RR. Can a shift in fuel energetics explain the beneficial cardioneral outcomes in the EMPA-REG OUTCOME study? A unifying hypothesis. Diabetes Care. 2016;39:1115-1122.
44.Aubert G, Martin OJ, Horton JL, Lai L, Vega RB, Leone TC, et al. The failing heart relies on ketone bodies as a fuel. Circulation. 2016;133:698-705. 